\title{
Essai de traitement électronique d'une constellation de manuscrits
}

De Mina de Vanghel à Rose et Vert (Stendhal)

Daniel Ferrer, Maria-Ignez Mena Barreto et Jean-Jacques Labia

\section{(2) OpenEdition}

\section{Journals}

Édition électronique

URL : http://journals.openedition.org/recherchestravaux/115

DOI : 10.4000/recherchestravaux. 115

ISSN : 1969-6434

\section{Éditeur}

UGA Éditions/Université Grenoble Alpes

Édition imprimée

Date de publication : 15 juin 2008

Pagination : 249-262

ISBN : 978-2-84310-125-0

ISSN : 0151-1874

\section{Référence électronique}

Daniel Ferrer, Maria-Ignez Mena Barreto et Jean-Jacques Labia, « Essai de traitement électronique

d'une constellation de manuscrits », Recherches \& Travaux [En ligne], 72 | 2008, mis en ligne le 15 décembre 2009, consulté le 03 février 2021. URL : http://journals.openedition.org/recherchestravaux/ 115 ; DOI : https://doi.org/10.4000/recherchestravaux.115 


\section{Essai de traitement électronique d'une constellation de manuscrits : de Mina de Vanghel à Rose et Vert (Stendhal)}

\section{Historique du projet}

Au commencement, il y avait le Centre d'études stendhaliennes et romantiques de l'Université Stendhal - Grenoble 3, associé au CNRS, département des Sciences de l'Homme et de la Société dans le cadre du programme «Archives de la création» (I998-200I).

Il s'agissait alors de lancer le processus d'analyse et d'exploitation du fonds ensommeillé des manuscrits de Stendhal et d'imaginer de nouvelles manières de traiter les documents de genèse. Alors, la Bibliothèque de Grenoble était déserte et vide. Gérald Rannaud choisit pour commencer, à titre d'exemple, le dossier génétique d'une ébauche de roman non pas abandonnée, mais laissée inachevée par Stendhal, Le Rose et le Vert, commencée le I 8 avril I 837 à Paris et continuée jusqu'aux premiers jours de juin, lors du séjour à Nantes, où Stendhal est en repérages pour les Mémoires d'un touriste. Le processus d'écriture est alors suspendu, et non pas abandonné, puisqu'une page écrite à la veille même de la mort de Stendhal, le 2 I mars I 842, sous le titre La Juive, prolonge et réoriente ce projet, qu'elle clôt de fait par death of the author. Malgré la discontinuité chronologique et la différence de genre, ce dossier n'est pas sans rapport avec un texte antérieur, celui d'une nouvelle achevée, sous le titre 
Mina de Vanghel, écrite en décembre I 829 et janvier I 830, corrigée en I 832 , inédite du vivant de l'auteur. Le choix était motivé par la richesse et la complexité, rares dans le fonds stendhalien, avec trois états du texte, sans compter le lien problématique avec le manuscrit incomplet et le texte posthume de la nouvelle dans la Revue des deux Mondes.

Une première équipe fut alors constituée avec un pôle grenoblois (Gérald Rannaud, Serge Linkès) et un pôle parisien (ITEM, Daniel Ferrer). Une entreprise parallèle venait alors d'aboutir à la publication en Garnier-Flammarion, sous le titre Le Rose et le Vert, du texte de la nouvelle Mina de Vanghel revu d'après le manuscrit, associé à l'ensemble du dossier de Rose et Vert, selon le principe d'une réunion des deux textes de fiction constituant la matière allemande de Stendhal, autour d'une hérö̈ne nommée Mina. Jean-Jacques Labia, éditeur de ce volume, rejoignit l'équipe, ainsi que Maria-Ignez Mena Barreto, qui préparait alors sa thèse sur le Brulard ${ }^{\mathrm{I}}$.

\section{Description du corpus}

Le corpus au sens strict commence par un manuscrit originel, autographe, occulté et oublié, redécouvert depuis peu, Tamira Wanghen. Gérald Rannaud avait rencontré l'une de ses sept pages curieusement réemployée au verso en guise de page de titre dans le manuscrit du Brulard, et donné une transcription commentée de l'ensemble dans la revue $H B^{2}$, parallèlement à la publication de cet inédit dans le dossier de l'édition GF par Jean-Jacques Labia (tous deux en I998). C'est le manuscrit I. Il a été conservé par Stendhal parce qu'il reste incomplètement exploité dans les états suivants. Il comporte en effet une bifurcation quant à l'identité de l'héroïne (initialement juive allemande, puis allemande non juive en I 837), sur laquelle reviendra, pour une réorientation interrompue par la mort de Stendhal, la page ultime du dossier (La Juive, en I 842). Le manuscrit principal, le plus complet (tantôt de la main d'un copiste, et tantôt autographe), livre l'état final, inachevé, avec des plans et des scénarios pour la suite : c'est le manuscrit 3. Par un hasard heureux, un deuxième état fragmentaire (copiste corrigé) nous est également parvenu et vient encore enrichir le dossier : c'est le manuscrit 2.

Restait à poser la question du rapport à un autre document (manuscrit o) constitué par un manuscrit commencé en décembre i 829 et terminé le 7 janvier

I. M.-I. Mena Barreto, «La construction d'une représentation de soi dans le manuscrit de La Vie de Henry Brulard: essai d'approche génétique», thèse de Doctorat sous la direction de D. Ferrer, Université Paris VIII, 2005.

2. G. Rannaud, «Tamira Wanghen et les limites du récit», $H B, \mathrm{n}^{\circ}$ 2, I 998, p. 37-5 I. 
I 830 pendant la genèse de Rouge et Noir, celui de la nouvelle Mina de Vanghel, manuscrit autographe, incomplet, relu et corrigé deux fois, immédiatement en I 830, et de nouveau en I 832, à compléter par le texte de l'édition originale posthume publié par Romain Colomb dans la Revue des deux Mondes. Il ne peut en effet être écarté, même si Stendhal n'en dispose pas lorsqu'il se lance dans le projet du Rose et Vert, en raison de la note («Préface confidentielle à Ko», destinée à Colomb) qui envisage dès I 830 l'amplification de la nouvelle sous forme de roman en deux volumes sur le modèle contemporain (en I 830) de Rouge et Noir, et qui donne sans doute la raison principale de sa non publication:

Décrire les mœurs est froid dans un roman

c'est presque moraliser. Tournez la description en

étonnement, mettez un étranger qui s'étonne; la

description devient un sentiment. Le lecteur a

quelqu'un avec qui il peut sympathiser

Mina étrangère d'un pays

Julien, jeune provincial élève

$$
\text { où l'on philosophie }
$$

de Plutarque et de Napoléon,

Introduire dans Mina if enflée à 2 vol le jeune homme fort ennuyé

marquis et libéral voulant aimer l'amérique

et le moyen-âge pendant lequel brillait sa famille et laconiquement triste

Par la suite la nouvelle sert, nous le verrons, de carrière virtuelle de réemploi. On aura donc dans cette hypothèse : un état 0 (absent, présent, voire encombrant), un état I (Tamira Wanghen), un état 2 (intermédiaire, corrections de I intégrées, copiste corrigé), un état 3 (copiste, corrections de 2 intégrées, autographe). À cela s'ajoutent un certain nombre de plans, récapitulatifs ou projectifs, qui constituent autant de versions d'époques et de portée diverses, mais qu'on groupera par commodité sous le chiffre 4 .

\section{Une aporie génétique}

L'exploitation de cette constellation de manuscrits présente un certain nombre de difficultés pour une approche génétique de type classique, en particulier l'état lacunaire des manuscrits tels qu'ils nous sont parvenus. En ce qui concerne le manuscrit de la nouvelle La vie et la mort de Mina de Vanghel, un examen même superficiel du manuscrit montre qu'il s'agit d'un manuscrit composite, ayant subi des remaniements de taille, avec découpage, déplacement, collage, ajout et suppression d'un nombre important de feuillets. L'étude codicologique du dossier corrobore cette première impression. Le deuxième ensemble de manuscrits - le dossier du Rose et Vert - est un peu plus fourni, 
puisque nous disposons d'un premier jet et d'un état intermédiaire en plus d'un dernier état très remanié bien qu'inachevé. Mais Tamira Wanghen, le premier jet, s'arrête au bout de sept pages et il manque le début de la copie intermédiaire qui par ailleurs s'interrompt au moment où Mina quitte l'Allemagne, si bien que nous ne sommes à même d'établir des comparaisons directes que sur un étroit segment des versions I à 3 .

À cette première difficulté vient s'ajouter le caractère relativement lâche et distendu qui caractérise les rapports génétiques qu'entretiennent les deux groupes de manuscrits. Bien qu'apparentés sous de nombreux aspects, nous avons ici affaire à deux projets littéraires distincts. Le manuscrit de Mina de $V$ anghel correspond à un état sinon définitif, en tout cas très avancé d'un projet de nouvelle qui a dans le contexte historique et éditorial de I 829-I 830 ses raisons d'être et ses motivations spécifiques. Sa publication par Romain Colomb dans la Revue des deux Mondes en 1853 témoigne de sa tenue et de sa valeur en tant qu'œuvre littéraire à part entière : il serait de toute évidence abusif de réduire ce manuscrit au statut d'un simple antécédent génétique du roman entamé en I 837.

Quant au manuscrit du Rose et Vert, sa rédaction ne semble avoir été que partiellement et indirectement déterminée par l'expérience de la nouvelle. Plusieurs indices corroborent l'hypothèse selon laquelle Stendhal n'avait pas en I 837 le manuscrit de Mina de Vanghel sous la main. Sur la page 7 du manuscrit de Tamira Wanghen, on lit en effet la note :

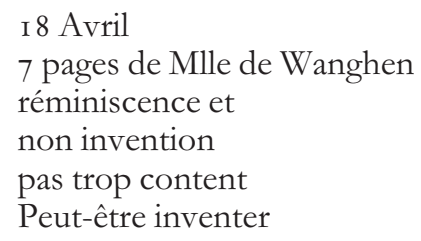

puis, sur le verso de la page i 6 de la copie du i 9 avril, la note restée longtemps énigmatique, que nous croyons avoir déchiffrée :

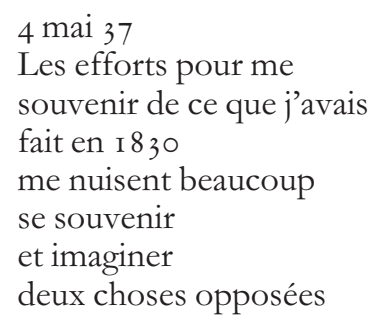

Stendhal se souvient en l'absence du manuscrit de la nouvelle, et ceci presque malgré lui. Ces deux notes apportent la preuve d'un fait déjà fortement suggéré par la confrontation de deux dossiers : il n'y a pas eu remploi ou 
réutilisation directe du manuscrit de Mina de Vanghel dans la rédaction du Rose et Vert. Ceci nous interdit de poser les rapports qui se tissent entre la nouvelle et le roman dans les termes d'une réécriture classique. Et par conséquent, de procéder à une mise en parallèle pour confrontation pièce à pièce des différentes versions successives, comme le veut la méthode héritée de la philologie classique traditionnellement en usage parmi les généticiens (moins pour mettre en paradigme des «lieux variants» que pour repérer le sens global d'une évolution).

Or, bien que la filiation entre les deux projets ne passe pas par le canal habituel de la réécriture, ces deux ensembles entretiennent néanmoins des relations génétiques suffisamment fortes pour que nous soyons obligés de les traiter conjointement. L'idée du Rose et Vert, nous l'avons vu, nait dans les brouillons de Mina de Vanghel. À en croire la préface confidentielle à Colomb citée plus haut, l'idée «d'enfler» le texte de la nouvelle à un roman en deux volumes n'est postérieure à la rédaction des dernières pages de Mina de Vanghel que d'une quinzaine de jours. Et cette idée n'est sans doute pas sans rapport avec la mise en réserve du manuscrit et la décision de ne pas le publier à une époque où Stendhal s'essaie pourtant au genre de la nouvelle.

Une comparaison un tant soit peu systématique des deux ensembles manuscrits nous permet de repérer la circulation d'éléments aux statuts divers - thèmes, figures, scènes, personnages, lieux, etc. - qui, de la nouvelle aux derniers plans, sont repris, développés, déplacés, transformés, abandonnés, puis à nouveau réintégrés de l'un à l'autre manuscrit. Tout se passe comme si, lors de la rédaction de la nouvelle en i 829-1 830 , un premier ensemble d'éléments narratifs, des situations inventées de toutes pièces ou puisées dans un fonds de souvenirs personnels, parfois littéraires - bref, tout un répertoire de motifs, s'était cristallisé autour d'un premier argument et s'était progressivement ordonné à l'intérieur d'une structure de séquence, d'un syntagme stable et cohérent. Ne connaissant que la dernière phase de ce processus, nous ne pouvons dans le meilleur des cas qu'émettre des hypothèses quant aux aléas de son déroulement effectif. Ce même répertoire, ou plutôt ce que Stendhal en avait conservé dans sa mémoire, a été repris dans le cadre du projet de roman de I 837, redistribué, développé, certains éléments ayant été abandonnés définitivement ou parfois repris sur de nouvelles bases au fil des versions et plans successifs. Le travail de réécriture mené dans le cadre de ce deuxième projet lui-même s'est fait de façon discontinue et non linéaire : Stendhal semble ici avoir procédé par retouches, par amplification de certaines séquences ponctuelles, alors que d'autres séquences contiguës passent pratiquement à l'identique d'une version à l'autre. Le manuscrit se dilate progressivement, mais par boursouflures localisées. De sorte que la configuration générale de cet ensemble de manuscrits ressemble davantage à un amas 
nébuleux en expansion qu'à un parcours évolutif se déployant de façon relativement uniforme et régulière. De même que le personnage de Mina/Tamira cherche sa vérité dans l'exil, prenant soin de ménager des fausses pistes et prenant le risque d'une défiguration provisoire, de même les thèmes principaux sont engagés dans une migration systématique, se déformant pour s'ajuster en des figures mouvantes.

\section{Matériaux}

Pour travailler sur cet ensemble, nous disposions, grâce à l'équipe grenobloise, d'images numériques des manuscrits, d'une superbe transcription diplomatique des versions I et 2 et des premières pages de la version 3, réalisée par Yvonne et Gérald Rannaud, et d'un stemma établi par Gérald Rannaud, clarifiant les relations entre les différentes parties du dossier. Nous disposions par ailleurs d'une transcription linéarisée de la totalité du dossier et d'une analyse thématique préliminaire, réalisées par Jean-Jacques Labia (voir fig. I, infra, p. 255$)$.

\section{Constitution d'un système de bases de données}

Puisqu'il nous était impossible de définir un fil conducteur homogène sur lequel émergeraient les différences entre les quatre «versions» (sans oublier les variantes internes de chacune d'entre elles) et les différents plans, nous nous trouvions dans l'incapacité de superposer des versions isomorphes. Il n'était cependant pas question de renoncer à la comparaison d'états, qui est évidemment essentielle pour définir un processus d'écriture, mais on ne pouvait se contenter de la juxtaposition de quelques fragments intuitivement choisis, comme dans la traditionnelle «méthode des passages parallèles». Nous avons donc décidé de voir si un système de base de données relationnelles pouvait nous aider à procéder de manière moins impressionniste et plus systématique, tout en sachant bien qu'un tel dispositif ne nous restituerait rien d'autre que ce que nous y aurions introduit et que la subjectivité qui se glisserait dans la préparation des données ne serait pas atténuée du fait qu'elle se dissimulerait, dans la présentation finale, sous l'apparente objectivité d'un traitement machinique. Nous avions toutefois l'espoir, qui s'est révélé en partie justifié, que la tabulation des résultats mettrait en évidence une partie des manques et des points aveugles, et que la souplesse de la base (FileMaker ${ }^{\circledR}$ Pro) nous permettrait de corriger les données et de rectifier la structure au fur et à mesure du traitement et de l'exploitation. 
ESSAI DE TRAITEMENT ÉLECTRONIQUE D’UNE CONSTELLATION DE MANUSCRITS...

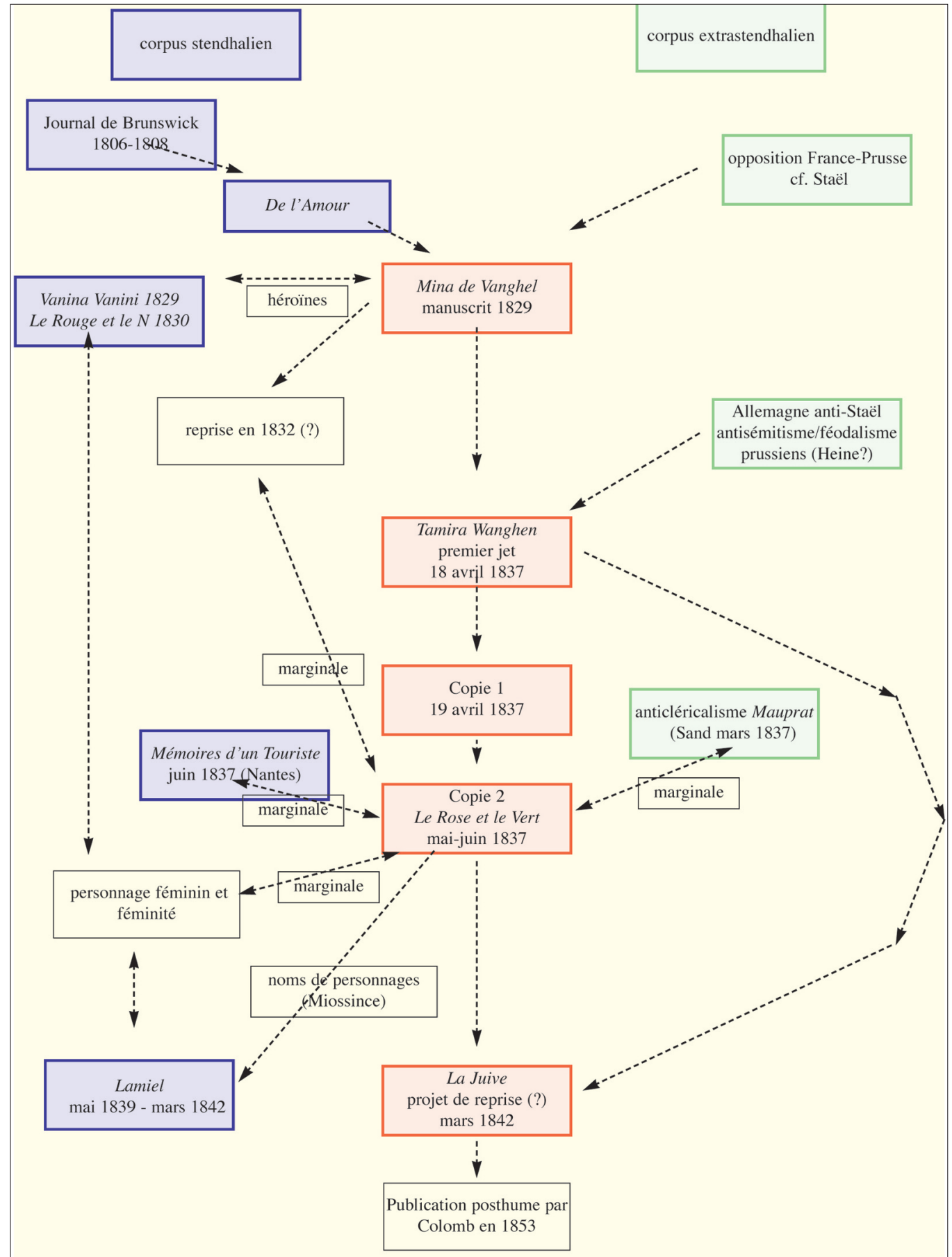

Fig. I. Stemma Rose et Vert. 


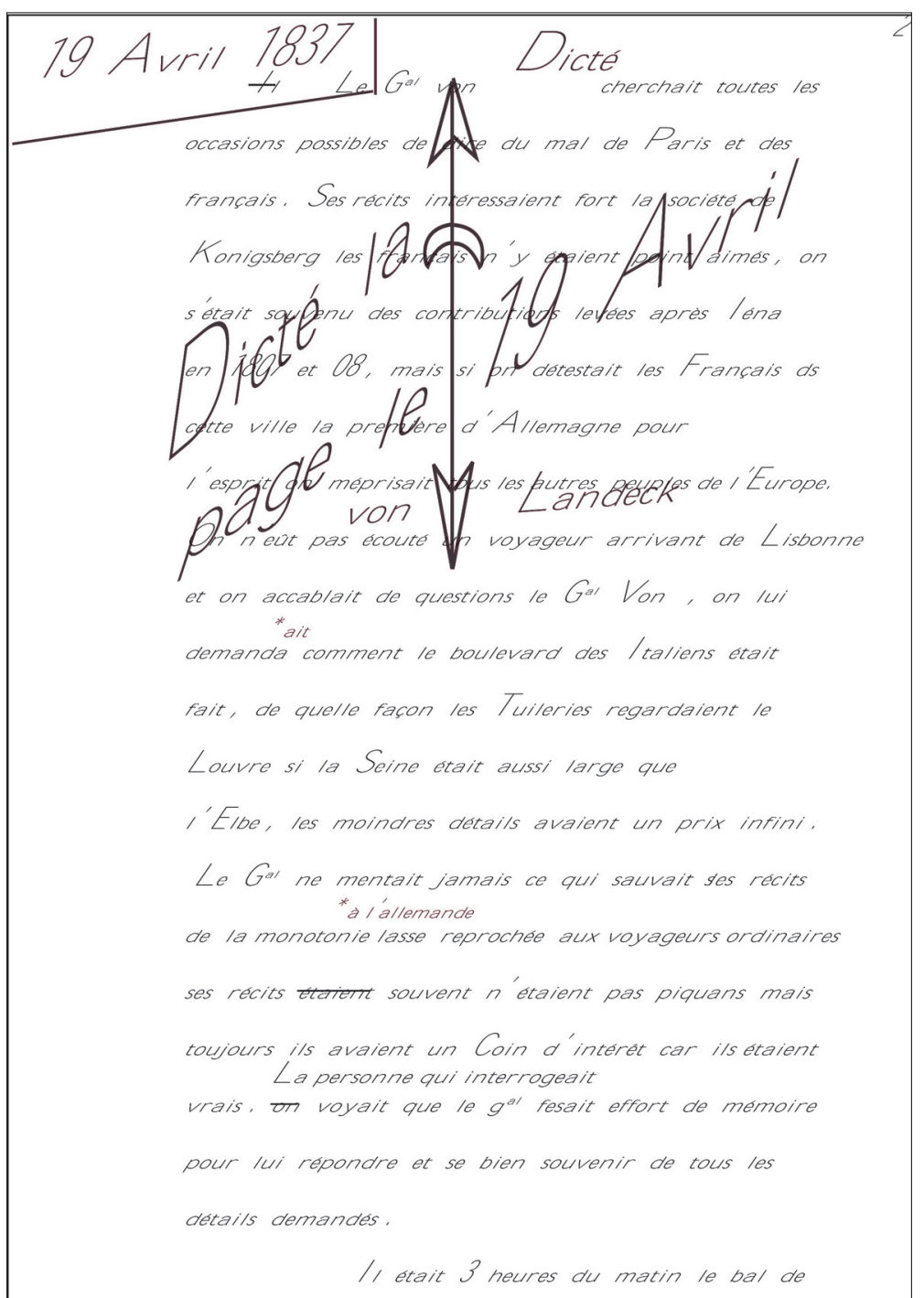

Fig. 2. Une page de transcription.

Nous avons découpé l'ensemble du matériau étudié en segments d'ampleur variable, que nous avons rangés dans une base nommée «Lexies», en hommage à Roland Barthes. Comme chez lui, «le morcellement du texte narratif en lexies est purement empirique, dicté par un souci de commodité : la lexie est un produit arbitraire, c'est simplement un segment à l'intérieur duquel on observe la répartition du sens ${ }^{3} »$. Mais contrairement aux lexies de Barthes dont le découpage était calculé pour rassembler deux ou trois sens chacune, les nôtres renvoient le plus souvent à une dizaine d'unités de signification. 
Chaque lexie est reliée à son support matériel par la cote de la page manuscrite où elle figure (ou exceptionnellement par la référence à la page de la Revue des Deux Mondes pour les passages de Mina de Vanghel qui manquent dans le manuscrit) et affectée d'un numéro de version ( 0 pour Mina de Vanghel, I pour Tamira Wanghen, 2 pour la copie intermédiaire, 3 pour Le Rose et le Vert, 4 pour les plans), avec un système de + et de - pour les variantes notables de chacun des états (par exemple, si un passage de Tamira Wanghen comporte des ratures et des additions, on distinguera une version I-, avant ratures, une version I, après ratures, et une version $\mathrm{I}+$, après additions). Ces variantes internes à chaque document sont indiquées dans le texte même par un code de couleurs.

\section{Les noyaux sémantiques}

Nous avons attribué à chacune de ces lexies un certain nombre de «sèmes» ou noyaux sémantiques. Plus que dans le découpage des lexies, c'est évidemment dans la nomination des sèmes et dans leur attribution qu'une grande part de subjectivité interprétative intervient inévitablement. Pour l'atténuer, nous n'avons pas hésité à multiplier ces étiquettes de portée et de pertinence très diverses, au prix d'une certaine redondance : par exemple «circulation nord-sud» coexiste avec «circulation France-Allemagne» ou «banquier» avec «richesse». On ne saura qu'a posteriori quel découpage se révèle le plus productif, et, en attendant, le coût de cette redondance est faible.

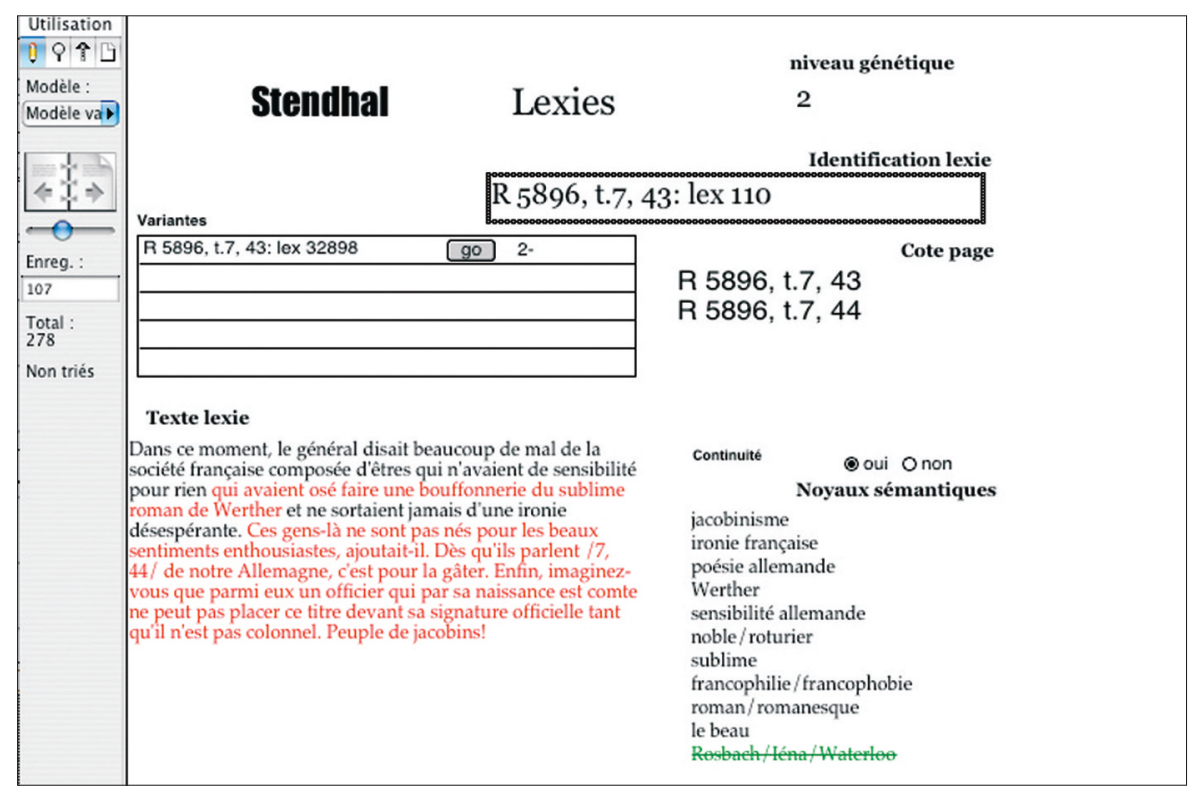

Fig. 3. Lexie. 


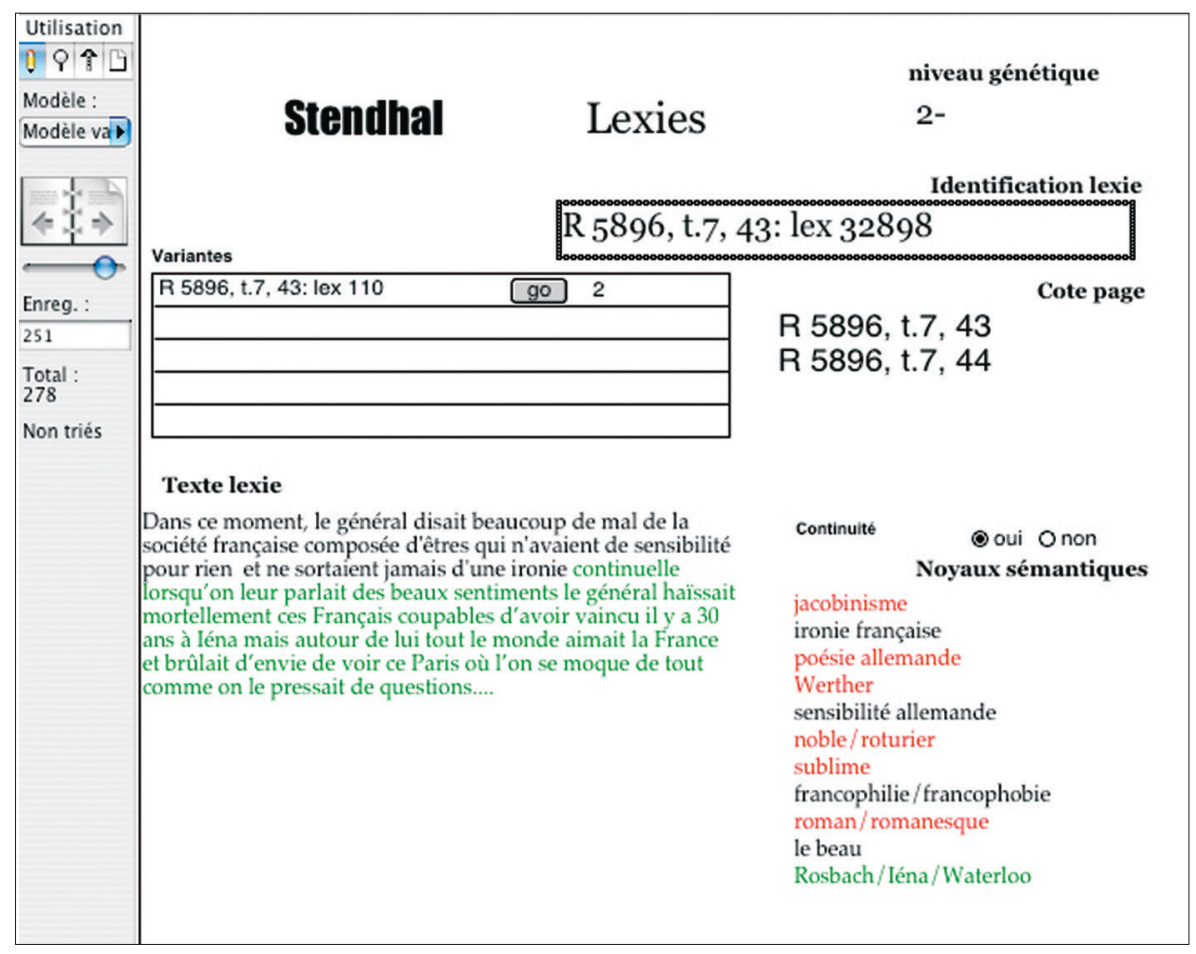

Fig. 4. Lexie.

Une fois ce travail (assez long) effectué pour l'ensemble du corpus, cette première base est reliée à deux autres, interrogeables séparément, qui reprennent la liste des sèmes (base NS) et la liste des documents (base Pages). Il est dès lors facile de faire apparaitre dans une table la totalité des occurrences de chaque sème, classées par version, incluant ou non la version 0 ou la «version» 4, avec dans chaque cas la liste des sèmes co-occurrents. Le parallélisme d'ensemble qui nous manquait pour saisir le mouvement de la genèse est remplacé par un parallélisme partiel mécaniquement constitué, qui permet de saisir la migration des éléments, la circulation des noyaux sémantiques d'une version à l'autre. À partir de là, toute l'interprétation reste à faire, mais celle-ci se trouvera facilitée par cette mise en paradigme automatisée. Précisons qu'il ne s'agit absolument pas d'une concordance lexicale, car les thèmes peuvent se dissimuler sous les signifiants les plus divers.

Ainsi, pour prendre un exemple simple, si nous observons la table des occurrences du sème «banquier», nous voyons du premier coup d'œil qu'il est absent de 0 (la nouvelle est antérieure à la Monarchie de juillet et au rôle accru des banquiers dans la réalité politique comme dans l'imaginaire littéraire). 
Dans I, il est associé à la judéité, mais cette association disparaît à partir de $\mathrm{I}^{+}$, quand Salomon Wanghen devient Pierre Wanghen. Elle réapparait de manière floue dans 3, avec le Comte de Vintimille, et implicitement dans 4, avec La Juive .

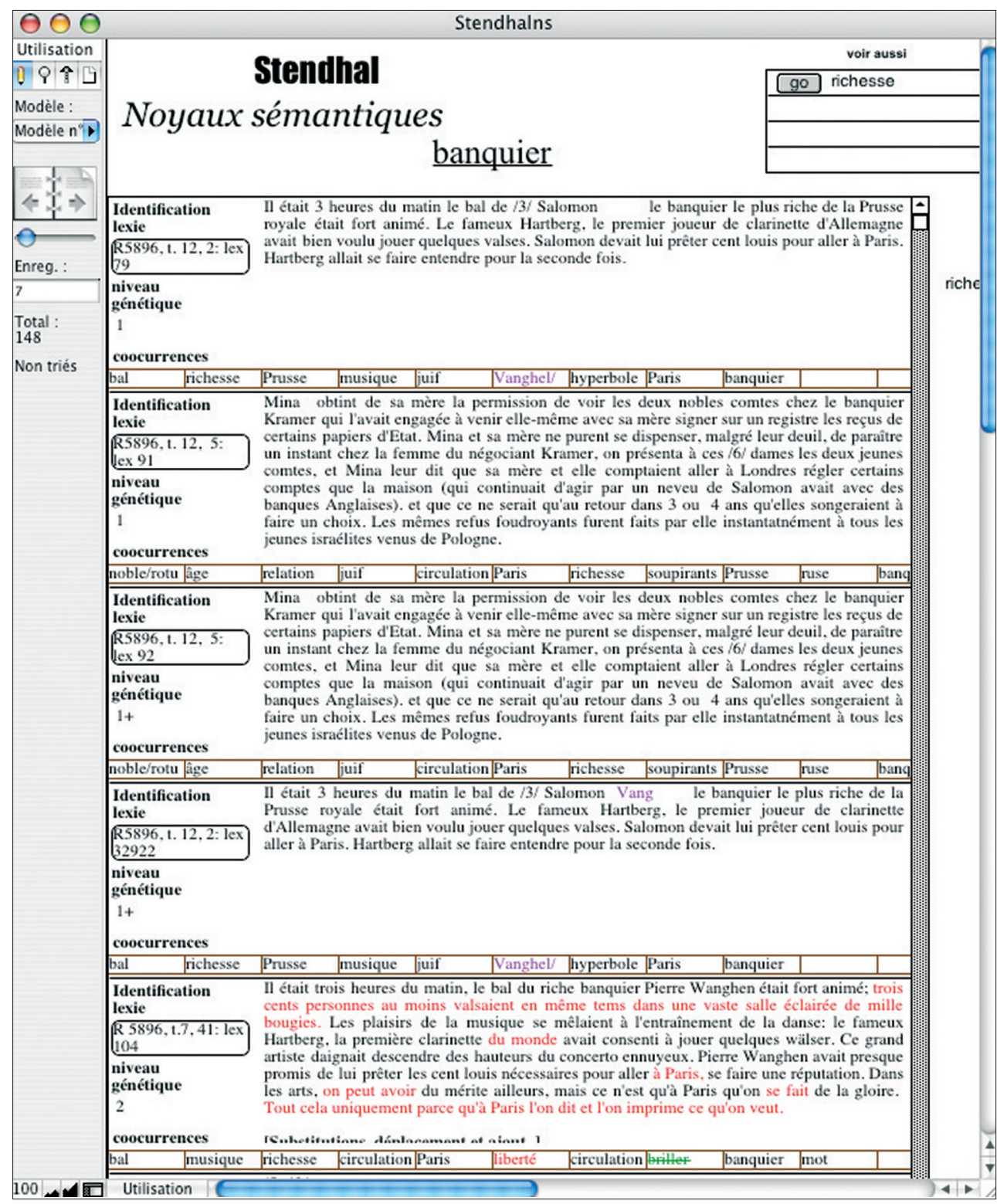

Fig. 5. Vue partielle de la table «banquier». 
Si nous examinons la table «bavard» nous voyons que dans 0 , ce sème est attribué tardivement à M. de Ruppert, tandis que dans I, 2 et 3 ainsi que dans la plupart des plans (niveau 4), le bavardage apparait dès le début, associé comme une épithète homérique au Général von Landeck, sorte de figure auto-ironique de l'auteur, barbon aimant à se faire admirer des jeunes femmes et surtout opérateur de récit, de par sa prolixité. L'association est renforcée par des ajouts explicites aux niveaux I + et 2 .

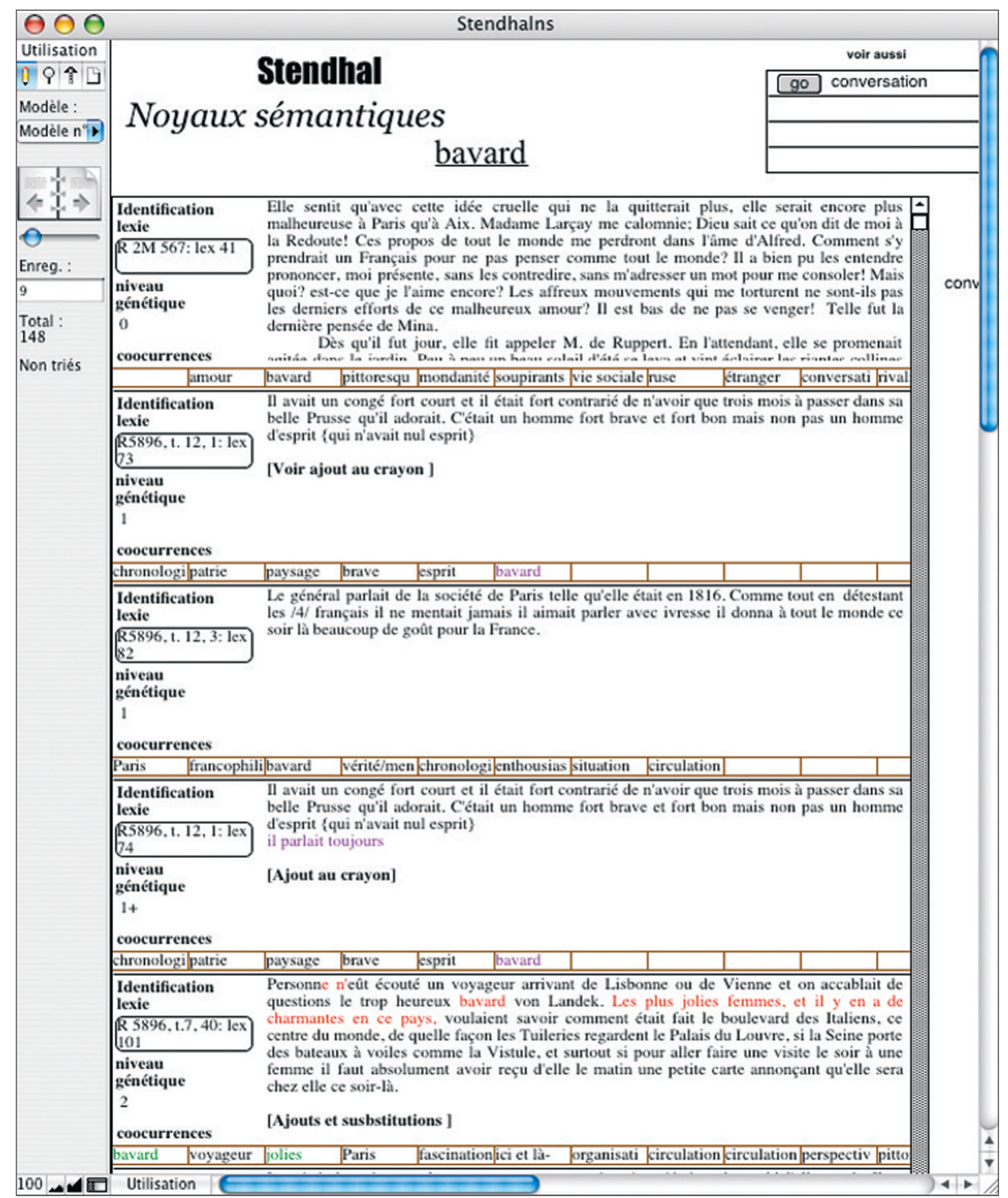

Fig. 6. Vue partielle de la table «bavard». 
ESSAI DE TRAITEMENT ÉLECTRONIQUE D’UNE CONSTELLATION DE MANUSCRITS...

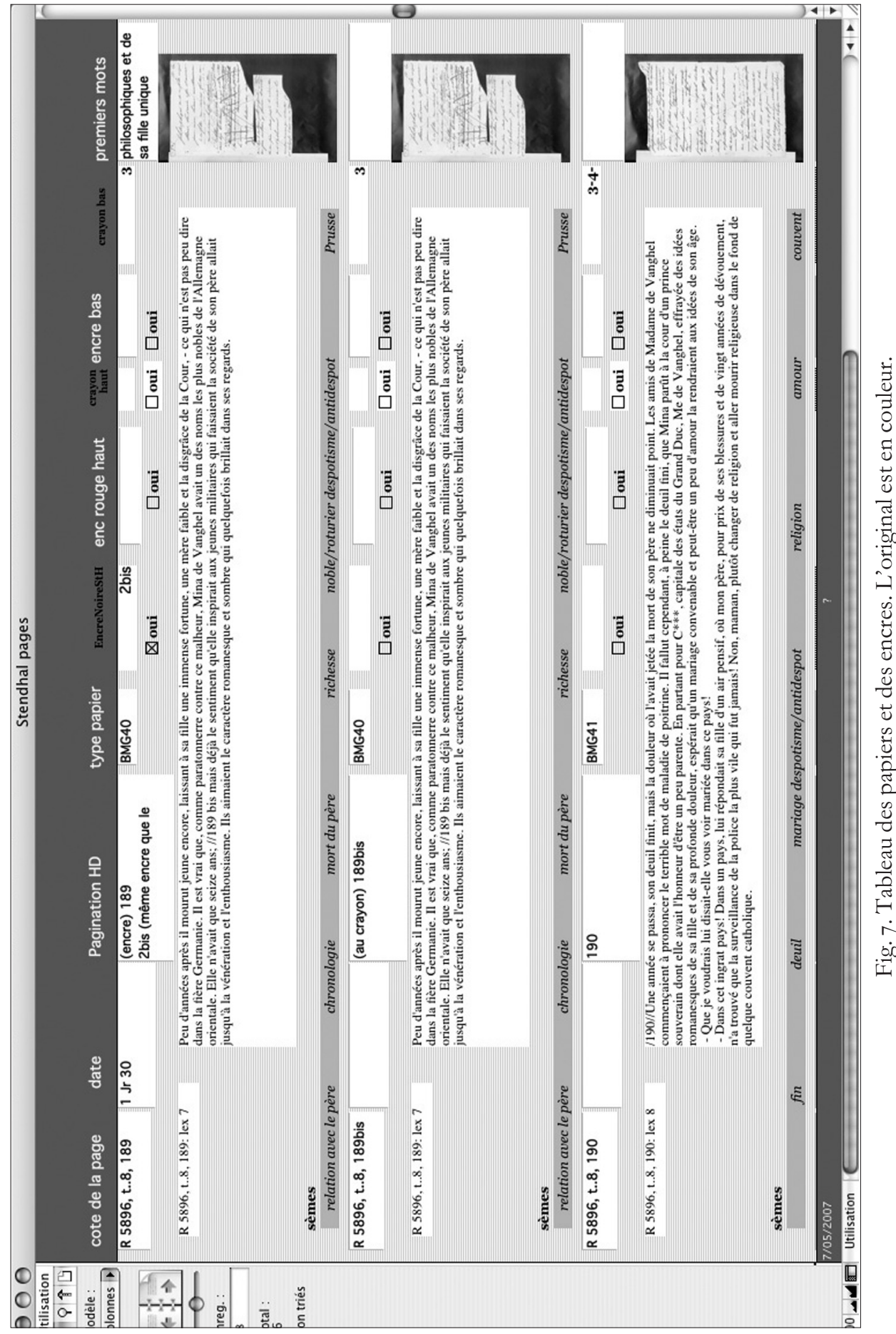




\section{Le support}

L'avantage d'un système de base de données ouvert, c'est qu'il permet d'associer ce découpage sémantique abstrait à toutes sortes d'autres données, et notamment aux données recueillies à la suite d'une étude matérielle des manuscrits. La tabulation des papiers, des encres et des inscriptions marginales permet par exemple de mettre en évidence plusieurs strates d'écritures dans la version 0 . En couplant la base «lexies» et la base "pages», il est possible d'effectuer des recoupements entre ces différentes strates et le contenu sémantique qu'elles véhiculent (voir fig. 7, supra, p. 26I).

\section{L'avenir}

Que faire de cette expérience? Pour le dire, il faudra sans doute continuer à utiliser la base pour produire des analyses génétiques. Seul l'usage déterminera si les solutions proposées peuvent servir de point de départ pour traiter toute une classe de dossiers de genèse caractérisés par la discontinuité. 